\title{
Evaluación del riesgo de introducción de peste equina africana a países del cono sur sudamericano por importación de animales vivos
}

\author{
Assessment of the risk for introduction of African horse sickness into \\ South American southern cone countries via import of live animals \\ A Marcos ${ }^{\mathrm{a}}, \mathrm{M}$ Roco $^{\mathrm{b}}, \mathrm{C}$ Picasso ${ }^{\mathrm{c}}, \mathrm{L}$ Ávila ${ }^{\mathrm{d}}, \mathrm{AM}$ Pérez $^{\mathrm{e}}$ \\ ${ }^{a}$ Dirección de Epidemiología y Análisis de Riesgo, Dirección Nacional de Sanidad Animal, \\ Buenos Aires, Argentina. \\ ${ }^{\mathrm{b}}$ Subdepartamento de Sanidad Animal, División de Proteccion Pecuaria, Servicio Agrícola y Ganadero, \\ Santiago, Chile. \\ ${ }^{\mathrm{c}}$ Departamento de Control de Comercio Internacional, División de Sanidad Animal, \\ Dirección General de Servicios Ganaderos, Montevideo, Uruguay.

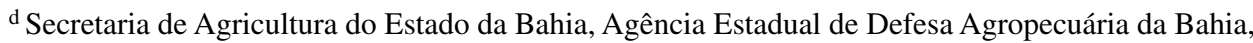 \\ Salvador de Bahia, Brasil. \\ e Department of Veterinary Population Medicine, College of Veterinary Medicine, University of Minnesota, \\ Saint Paul, Minnesota, USA.
}

\begin{abstract}
SUMMARY
African Horse Sickness (AHS) is arguably one of three animal diseases that are exotic to countries in the Southern Cone of South America and that, if introduced into the region, would result in substantial economic and social consequences. This study shows the results of a quantitative risk assessment of the introduction of AHS into the region through the legal importation of horses from Europe, under the hypothesis of an undetected epidemic in the country of origin. Results suggest that the risk for introduction of AHS in the scenario assessed here is low, and that in the event of an epidemic in the country of origin, for those animals that are in transit when the epidemic is detected and given that the farm of origin was certified negative, a negative serological test in destination may be sufficient to lower the risk for introduction down to acceptable levels. These results also suggest that current legislation in the region is sufficient for preventing the introduction of the disease.
\end{abstract}

Key words: risk assessment, African horse sickness, South America.

\section{RESUMEN}

La Peste Equina Africana (PEA) es una de las tres enfermedades exóticas al cono sur sudamericano que ha sido identificada como potencial generador de cuantiosas pérdidas económicas y sociales en caso de que se introduzca en la región. Este trabajo presenta los resultados de un análisis de riesgo cuantitativo de introducción de PEA en la región por medio de la importación legal de equinos vivos de Europa y bajo el supuesto de que ocurra una epidemia no detectada en el país de origen y durante la fase silente de la epidemia. Los resultados sugieren que el riesgo de que se produzca tal evento es bajo y que, en caso de una epidemia en el país de origen, para aquellos animales que se encontraban en tránsito al momento de la detección y debido a que el establecimiento de origen permanece negativo, una prueba serológica negativa podría ser suficiente para mitigar el riesgo de introducción. Estos resultados sugieren también que la legislación vigente en la región y debido a las condiciones epidemiológicas actuales, es suficiente para prevenir el ingreso de PEA.

Palabras clave: evaluación de riesgo, peste equina africana, Sudamérica.

\section{INTRODUCCIÓN}

La Peste Equina Africana (PEA) es una enfermedad infecciosa viral que afecta a los équidos, transmitida por vectores biológicos, principalmente del género Culicoides (Mellor 1993). El virus de la PEA (VPEA) pertenece a la familia Reoviridae y al género Orbivirus, habiéndose descrito nueve serotipos del VPEA (Scacchia y col 2009,

Aceptado: 03.07.2014.

* 335A Animal Science Veterinary Medicine Building, 1988 Fitch Ave, St. Paul, MN 55108, USA; aperez@umn.edu
MacLachlan y Guthrie 2010). La PEA es endémica en ciertas regiones de África, mientras que se han reportado epidemias aisladas en Egipto, España, Portugal, Pakistán y la India (Sánchez Vizcaíno 2004). La PEA es causante de grandes pérdidas económicas en la industria equina de regiones afectadas debido a la alta mortalidad de animales y a las restricciones del comercio y de eventos ecuestres a nivel internacional.

La primera referencia de esta enfermedad se remonta al año 1327 en Yemen, a partir de la introducción de equinos desde África (Mellor 1993, Mellor y Hamblin 2004). El virus permaneció endémico en África subsahariana por 
siglos y en el período 1959-61 el serotipo 9 se expandió fuera de África hacia varias regiones de Asia (Arabia Saudita, Siria, Líbano, Jordania, Irak, Turquía, Irán, Afganistán, Pakistán e India) y Chipre (Mellor 1993, Mellor y Hamblin 2004). En 1961, luego de una campaña de vacunación masiva y la muerte de 300.000 equinos, la enfermedad desapareció de Asia (Mellor 1993). En 1965 el serotipo 9 nuevamente se diseminó desde Marruecos, Argelia y Túnez hacia fuera de África, y se introdujo en España en octubre de 1966. La enfermedad se erradicó de España en tres semanas debido a una importante campaña de vacunación y a la política de sacrificio sanitario. Sin embargo, en el norte de África la PEA permaneció durante dos años. La aparición en esta zona se debió al movimiento nómada de personas y animales (especialmente burros) a través del Sahara. Por los siguientes 20 años la PEA solo se notificó en África subsahariana. En julio de 1987 se reportó un brote de PEA por el serotipo 4 en España (Mellor 1993). El origen de este brote fue una importación de cebras que cursaban la enfermedad de manera subclínica importadas desde Namibia. Los brotes continuaron en España durante 1988-1990, en Portugal en 1989 y en Marruecos en 1989-1991 (Mellor 1993). Todos estos brotes se debieron al serotipo 4, sugiriendo que el virus sobrevivió al invierno de la región mediterránea durante cinco años. En la actualidad la enfermedad se encuentra de forma endémica en la zona tropical y subtropical del África (MacLachlan y Guthrie 2010).

La PEA es una enfermedad exótica al continente americano y se considera que su ingreso a los países que integran el Comité Veterinario Permanente del Cono Sur (CVP) sudamericano (Argentina, Bolivia, Brasil, Chile, Paraguay y Uruguay) tendría efectos devastadores para la región. Esta apreciación fue el resultado de un trabajo conjunto realizado por técnicos designados por cada uno de los servicios veterinarios (SV) que integran el CVP realizado en junio del 2012 en la ciudad de Buenos Aires, Argentina. Cada técnico fue invitado a calificar a su país respecto de cada una de las enfermedades listadas por la Organización Mundial de Sanidad Animal (OIE) como "ausentes" o "confirmada o potencialmente presentes". Además, para aquellas enfermedades ausentes, se solicitó que cada país ponderara el impacto social y económico esperado en caso de sufrir una epidemia, donde 0 significaba ausencia de impacto sensible y 10 significaba un impacto superlativo. Tres enfermedades (influenza aviar, peste porcina africana y PEA) fueron declaradas como ausentes y con un impacto superlativo $($ media $=10)$ por los seis países participantes. Si bien la normativa de importación vigente en los países del CVP prohíbe el ingreso de animales y productos desde países o zonas infectadas con virus de VPEA, ciertamente, es posible la introducción de la enfermedad durante la fase silente de una epidemia desde zonas de las que sí se importa. Considerando además la historia reciente de epidemias de enfermedades equinas exóticas en países de la región, como Arteritis Viral Equina en la
República Argentina en $2010^{1}$, existe una percepción en los SV de que la introducción de la enfermedad a la región es posible. Por los motivos anteriormente expuestos, es necesario cuantificar el riesgo de introducción de PEA a los países del CVP y del impacto de potenciales medidas de mitigación del riesgo.

El objetivo de este trabajo consistió en determinar la probabilidad de introducción del VPEA a los países de la región mediante el movimiento de animales susceptibles. Se estima esta probabilidad en caso de que haya ocurrido una epidemia (reintroducción) de la enfermedad en el país de origen de los equinos. El presente trabajo constituye, además, el primer análisis de riesgo realizado en forma integral y conjunta por los países de la región.

\section{MATERIAL Y MÉTODOS}

\section{MODELO ANALÍTICO}

Se llevó a cabo un análisis de riesgo de tipo cuantitativo estocástico, utilizando metodologías analíticas recomendadas por la OIE (OIE 2013 a). Debido a que la normativa vigente en la región no permite el ingreso de animales desde países en los cuales esta enfermedad es prevalente, se consideró solo el riesgo asociado con movimiento de equinos desde Europa y en el supuesto de una reintroducción de esta enfermedad a Europa, durante el período silente. Se elige este continente debido a su historial de brotes de PEA y a que existe movimiento de animales susceptibles desde esta región hacia los países del CVP. Brevemente, se construyó un árbol de riesgo para el ingreso del VPEA a la región a través del movimiento legal de equinos vivos. El árbol consistió en cinco probabilidades independientes, específicamente:

- Probabilidad de que haya un establecimiento infectado (brote) no detectado en el origen (prevalencia predial durante la fase silente, $P P$ );

- Probabilidad de que un equino esté infectado en los establecimientos infectados no detectados (prevalencia animal, $P A)$;

- Probabilidad de que el equino infectado sobreviva a la enfermedad (probabilidad de supervivencia, $P S$ );

- Probabilidad de que no se detecte clínicamente el animal infectado (probabilidad de no detección clínica, PNDC)

- Probabilidad de que no se detecte serológicamente el animal infectado (probabilidad de no detección serológica, $P N D S$ )

La variable respuesta es la probabilidad de que al menos un animal infectado con VPEA ingrese a la región $\left(\mathrm{P}_{\mathrm{VPEA}}\right)$

www.oie.int/wahis_2/public/wahid.php/Reviewreport/Review?page_re fer $=$ MapFullEventReport\&reportid $=9233$. Fecha de consulta: 20 de diciembre de 2012. 
y se estimó como $\mathrm{P}_{\mathrm{VPEA}}=1$-[1-(PP x PA x PS x PNDC $\mathrm{x}$ PNDS) $]^{\mathrm{n}}$, donde $\mathrm{n}$ es el número de equinos ingresados.

\section{PARÁMETROS}

La incertidumbre y variabilidad asociados con las cinco probabilidades utilizadas en el modelo fueron cuantificadas utilizando una combinación de revisión bibliográfica y opinión de expertos. La revisión bibliográfica consistió en la consulta de artículos científicos y reportes de la OIE publicados en los últimos 10 años. Esta información fue utilizada para producir un listado de valores iniciales para cada probabilidad, la cual fue enviada a un experto de referencia, que no participó en el proceso de selección inicial de los parámetros, para que evaluara y eventualmente modificara estos valores en forma independiente. $\mathrm{La}$ información fue enviada al Centro de Vigilancia Sanitaria Veterinaria (VISAVET) de la Facultad de Veterinaria de la Universidad Complutense de Madrid que es uno de los cuatro laboratorios de referencia OIE para la enfermedad. Si bien el consultar a un único grupo de expertos conlleva un cierto riesgo de sesgo, debido al marco regulatorio de la OIE y el rol que juegan en ese marco sus centros de referencia, la opinión vertida por estos centros sirve de información de referencia objetiva que resulta en asistencia científica y técnica, así como asesoramiento sobre temas relacionados con la vigilancia y el control de la enfermedad ${ }^{2}$. Además se debe tener en cuenta que el mencionado centro se encuentra en España, un país con historia de epidemias de PEA, por lo cual dispone de la información empírica para evaluar la pertinencia de los valores asignados a cada probabilidad.

Para PP se asumieron tres escenarios determinísticos alternativos, correspondiendo, respectivamente, a valores de $\mathrm{PP}=0,01 ; 0,1 ; \mathrm{y} 1$. Estos valores corresponden a un mínimo de $1 \%$ de los establecimientos equinos infectados (un escenario realista basado en los datos históricos de cantidad de focos en España (Ministerio de Medio Ambiente, Medio Rural y Marino 2011) sobre el total de establecimientos (Ministerio de Agricultura, Pesca y Alimentación de España 2003)), un escenario intermedio que asume que la enfermedad se diseminó extensamente en el país exportador (10\% de establecimientos infectados) a efectos de ser conservadores en la estimación del riesgo $y$ un escenario extremo en que se asume que el establecimiento exportador está ciertamente infectado.

Utilizando datos de focos reportados a la OIE por Sudáfrica en 2006 y $2011^{3}$ y con la colaboración del experto consultado, para PA se asumió que este parámetro sigue una distribución lognormal con media $=0,32841$, desvío $=0,4058$ y shift $=-0,032947$, la que fue seleccionada

www.oie.int/es/nuestra-experiencia-cientifica/laboratorios-dereferencia/introducion. Fecha de consulta: 6 de mayo de 2013.

www.oie.int/wahis_2/public/wahid.php/Countryinformation/Countryreports. Fecha de consulta: 25 de octubre de 2012. debido a su buen ajuste a los datos empíricos, como sugiere el resultado de una comparación de valores observados y esperados mediante un test de Chi cuadrado $(\mathrm{P}=0,111)$. Debido a que la distribución lognormal puede arrojar valores incompatibles con las leyes de la probabilidad, la distribución fue truncada asumiendo como 0 y 1 a aquellos valores inferiores y superiores a los límites mínimos y máximo de la ley de la probabilidad, respectivamente.

Para PS, PNDC, PNDS se usaron distribuciones de tipo Pert, debido a la simplicidad de su formulación, que consiste en identificar, para cada probabilidad, tres valores (mínimo, más probable y máximo) a partir de los cuales se ajusta la distribución. Estos valores correspondieron a 0,36; 0,7 y 0,95 para PS, 0,01; 0,05 y 0,25 para PNDC y 0,$85 ; 0,97$ y 0,97 para PNDS. Los valores para PS se obtuvieron de los datos de las notificaciones realizadas a la OIE durante los años 2006 y 2011 de los países de África (Sudáfrica) y se calculó la letalidad (muertos/casos) de la enfermedad en cada foco ${ }^{4}$. Los valores de PNDC y más probable y máximo de PNDS fueron aportados por el laboratorio de referencia mencionado anteriormente, mientras que el mínimo de PNDS fue seleccionado considerando los valores de sensibilidad y especificidad reportados por la OIE y verificados por su laboratorio de referencia. Los valores de PA y PS son estimados basándose en la información obtenida de focos de Sudáfrica, debido a que no se disponen datos tan detallados de los focos ocurridos en Europa en los años 1966 y 1987 a 1990. Sin embargo, la decisión asumida fue ratificada por el centro de referencia OIE consultado.

Para n se consideraron cuatro escenarios, correspondientes al ingreso de 5, 10, 50 y 100 equinos durante el período silente de la epidemia, respectivamente. Estos cuatro escenarios fueron seleccionados considerando los datos de ingresos provistos por los servicios sanitarios de Argentina, Chile y Uruguay en los años 2011-2013. Uruguay ingresó cinco animales en 2012, mientras que el ingreso de equinos vivos a Chile en 2012 y a Argentina en 2013 desde países europeos fue de 127 y 93, respectivamente (servicios sanitarios de Argentina, Chile y Uruguay, comunicación personal). De esta manera, 5 y 100 son dos situaciones aproximadamente extremas en países de CVP, mientras que los valores de 10 y 50 representan dos escenarios intermedios. Se decidió mantener la estructura de escenarios, en lugar de ajustar una distribución que capture esta variabilidad, a los fines de que cada país pueda seleccionar el escenario que mejor refleje sus características demográficas y comerciales.

Todos los análisis fueron realizados utilizando el software @ RISK v. 5.7.1. (Palisade corporation, (22010), corriéndose 1000 iteraciones del modelo.

\footnotetext{
www.oie.int/wahis_2/public/wahid.php/Countryinformation/Countryreports. Fecha de consulta: 25 de octubre de 2012.
} 


\section{INDICADORES}

$\mathrm{El}$ indicador de interés fue el valor promedio de $\mathrm{P}_{\text {VPEA }}$ $\left(\mathrm{PP}_{\mathrm{VPEA}}\right)$ para cada uno de los 12 escenarios correspondientes a la combinación de tres valores de PP y cuatro valores de $\mathrm{n}$, los que fueron computados, alternativamente, asumiendo la exigencia de un resultado serológico negativo (como medida de mitigación) o la ausencia de realización de una prueba (correspondiente a la legislación actual). Valores de $\mathrm{PP}_{\mathrm{VPEA}}>0,01$ fueron considerados importantes, ya que sugieren que, en promedio, la región debería sufrir un brote de PEA por cada 100 brotes que ocurran en Europa.

El riesgo relativo asociado con la no realización de la prueba serológica (RRNPS) fue computado para cada uno de los 12 escenarios como el cociente entre el valor de $\mathrm{PP}_{\mathrm{VPEA}}$ estimado para la situación en que no se realiza prueba serológica dividido el valor de $\mathrm{PP}_{\mathrm{VPEA}}$ para la situación en que se exige un resultado serológico negativo. De esta manera, RRNPS es un indicador del beneficio esperado en caso de que se exija una prueba serológica negativa a animales importados a la región.

Además, se llevó a cabo un análisis de sensibilidad, mediante la estimación del coeficiente de correlación de Spearman (Rs) ajustado para controlar por correlación entre variables, para identificar los parámetros que más influyeron en el resultado.

\section{RESULTADOS Y DISCUSIÓN}

El riesgo de introducción de VPEA en el escenario realista (y conservadoramente alto) de $\mathrm{PP}=0,01$ fue bajo, no alcanzándose el nivel crítico de $\mathrm{PP}_{\mathrm{VPEA}}>0,01 \mathrm{ni}$ siquiera cuando se asumió la importación de 100 equinos durante la fase silente de la epidemia y en ausencia de un test serológico negativo confirmatorio (cuadro 1). Estos resultados son consistentes con la observación de que a pesar del frecuente movimiento de equinos desde Europa a América e incluso ante la ocurrencia de brotes en Europa, la PEA no ha sido jamás introducida en los países del cono sur sudamericano.

Probablemente, varios factores relacionados con la epidemiología de la enfermedad influyen en este resultado. Considerando los antecedentes mencionados en este trabajo, que son consistentes con el resultado del análisis de riesgo presentado aquí, estos sugieren que una combinación de alta mortalidad y condiciones muy restrictivas para la diseminación de la enfermedad se combinan para resultar en que la enfermedad se limite a África y que los brotes fuera de este continente sean limitados y poco frecuentes.

Los resultados de este trabajo sugieren que en un escenario de extensa diseminación de la enfermedad en el país exportador ( $\mathrm{PP}=0,1)$ la aplicación de un test serológico y su resultado negativo reduce el riesgo de introducción a niveles inferiores a los considerados como importantes en este estudio $\left(\mathrm{PP}_{\mathrm{VPEA}}<0,01\right)$ y en términos generales, el requerimiento de un test serológico negativo reduce en 15-20 veces el riesgo de introducción de la enfermedad en la región, como sugieren los valores estimados de RRNPS (cuadro 1). Un resultado serológico negativo también reduce significativamente el riesgo de introducción de PEA incluso ante la certeza de que el equino ha sido introducido desde un predio infectado $(\mathrm{PP}=1)$, siempre y cuando el número de equinos ingresados sea de 10 o menos. Este resultado es importante, porque, si bien la detección de un brote de PEA en un país importador a la región significaría la suspensión del comercio de animales y productos susceptibles (OIE $2013^{b}$ ), existe la posibilidad de que al momento de la detección hubiera animales en tránsito desde ese origen, cuya disposición genera, típicamente, controversia. Los resultados de este trabajo sugieren que en caso de ocurrencia de un

Cuadro 1. Probabilidad mediana (e intervalo de confianza del $95 \%$ ) de introducción de virus de la peste equina Africana (PP $\left.{ }_{\mathrm{VPE}}\right)$ en países del cono sur sudamericano y riesgo relativo asociado con la no realización de la prueba serológica (RRNPS), a partir del ingreso de equinos vivos desde Europa bajo la hipótesis de una epidemia no detectada en origen para 12 escenarios correspondiente a tres valores de probabilidad de granjas no detectadas en origen (PP) y cuatro números de animales importados por año (n). En negrita se indican valores de $\mathrm{PP}_{\mathrm{VPE}}>0,01$, que es compatible con la ocurrencia de una epidemia, en promedio, con una frecuencia superior a un brote cada 100 brotes en origen.

Median (95\% confidence interval) probability of African Horse Sickness virus introduction $\left(\mathrm{PP}_{\mathrm{VPE}}\right)$ into South American countries relative risk associated with non-performance of a serological test for horse movements from Europe under the hypothesis of an undetected epidemic in 12 scenarios, corresponding to 3 values of undetected farms probability (PP) and 4 numbers of imported animals per year (n). Values of $\mathrm{PP}_{\mathrm{VPE}}>0.01$, which are compatible with occurrence of an epidemic, on average, more frequently than once every 100 outbreaks, are indicated in bold.

\begin{tabular}{|c|c|c|c|c|c|c|c|c|c|c|c|c|}
\hline PP & 0,01 & & & & 0,1 & & & & 1 & & & \\
\hline $\mathrm{n}$ & 5 & 10 & 50 & 100 & 5 & 10 & 50 & 100 & 5 & 10 & 50 & 100 \\
\hline $\begin{array}{l}\mathrm{PP}_{\mathrm{VPEA}} \\
\text { con test } \\
\text { serológico } \\
\text { negativo }\end{array}$ & $\begin{array}{l}7,76 \times 10^{-6} \\
\left(3 \times 10^{-7} ;\right. \\
\left.5,94 \times 10^{-5}\right)\end{array}$ & $\begin{array}{l}1,55 \times 10^{-5} \\
\left(6 \times 10^{-7} ;\right. \\
\left.1,19 \times 10^{-4}\right)\end{array}$ & $\begin{array}{l}7,76 \times 10^{-5} \\
\left(3 \times 10^{-6}\right. \\
\left.5,94 \times 10^{-4}\right)\end{array}$ & $\begin{array}{l}1,55 \times 10^{-4} \\
\left(6 \times 10^{-6} ;\right. \\
\left.1,19 \times 10^{-3}\right)\end{array}$ & $\begin{array}{l}7,76 \times 10^{-5} \\
\left(3 \times 10^{-6} ;\right. \\
\left.5,94 \times 10^{-4}\right)\end{array}$ & $\begin{array}{l}1,55 \times 10^{-4} \\
\left(6 \times 10^{-6}\right. \\
\left.1,19 \times 10^{-3}\right)\end{array}$ & $\begin{array}{l}7,76 \times 10^{-4} \\
\left(3 \times 10^{-5}\right. \\
\left.5,93 \times 10^{-3}\right)\end{array}$ & $\begin{array}{l}1,55 \times 10^{-3} \\
\left(6 \times 10^{-5} ;\right. \\
\left.1,18 \times 10^{-2}\right)\end{array}$ & $\begin{array}{l}7,76 \times 10^{-4} \\
\left(3 \times 10^{-5}\right. \\
\left.5,93 \times 10^{-3}\right)\end{array}$ & $\begin{array}{l}1,55 \times 10^{-3} \\
\left(6 \times 10^{-5}\right. \\
\left.1,18 \times 10^{-2}\right)\end{array}$ & $\begin{array}{l}7,73 \times 10^{-3} \\
\left(3 \times 10^{-4}\right. \\
\left.5,77 \times 10^{-2}\right)\end{array}$ & $\begin{array}{l}1,54 \times 10^{-2} \\
\left(6 \times 10^{-4}\right. \\
0,112)\end{array}$ \\
\hline $\begin{array}{l}P_{\text {VPEA }} \\
\text { sin test } \\
\text { serológico } \\
\text { RRNPS }\end{array}$ & $\begin{array}{l}1,64 \times 10^{-4} \\
\left(6,43 \times 10^{-6}\right. \\
\left.1,19 \times 10^{-3}\right) \\
21,18\end{array}$ & $\begin{array}{l}3,29 \times 10^{-4} \\
\left(1,29 \times 10^{-5}\right. \\
\left.2,38 \times 10^{-3}\right) \\
21,18\end{array}$ & $\begin{array}{l}1,64 \times 10^{-3} \\
\left(6,43 \times 10^{-5}\right. \\
\left.1,18 \times 10^{-2}\right) \\
21,16\end{array}$ & $\begin{array}{l}3,28 \times 10^{-3} \\
\left(1,29 \times 10^{-4}\right. \\
\left.2,36 \times 10^{-2}\right) \\
21,15\end{array}$ & $\begin{array}{l}1,64 \times 10^{-3} \\
\left(6,43 \times 10^{-5}\right. \\
\left.1,19 \times 10^{-2}\right) \\
21,17\end{array}$ & $\begin{array}{l}3,28 \times 10^{-3} \\
\left(1,29 \times 10^{-4}\right. \\
\left.2,36 \times 10^{-2}\right) \\
21,15\end{array}$ & $\begin{array}{l}1,63 \times 10^{-2} \\
\left(6,43 \times 10^{-4}\right. \\
0,11) \\
21,02\end{array}$ & $\begin{array}{l}3,23 \times 10^{-2} \\
\left(1,29 \times 10^{-3}\right. \\
0,21) \\
20,86\end{array}$ & $\begin{array}{l}1,63 \times 10^{-2} \\
\left(6,43 \times 10^{-4}\right. \\
0,11) \\
21,05\end{array}$ & $\begin{array}{l}3,24 \times 10^{-2} \\
\left(1,29 \times 10^{-3}\right. \\
0,21) \\
20,88\end{array}$ & $\begin{array}{l}0,15 \\
\left(6,41 \times 10^{-3}\right. \\
0,7) \\
19,63\end{array}$ & $\begin{array}{l}0,28 \\
\left(1,28 \times 10^{-2}\right. \\
0,91) \\
18,21\end{array}$ \\
\hline
\end{tabular}


brote en un país europeo y en caso de que se encuentren animales en tránsito al cono sur sudamericano al momento de detectarse la enfermedad, una combinación de inspección clínica, comprobación de ausencia de infección en la granja de procedencia y un test serológico negativo podrían ser medidas de mitigación suficientes.

El modelo aquí ajustado fue relativamente robusto y solo influenciado significativamente $(\mathrm{P}<0,05)$ por los valores de PA $(\mathrm{Rs}=0,83)$ y PNDC $(\mathrm{Rs}=0,40)$. Este resultado demuestra la importancia de la detección temprana y reporte inmediato en el marco de la prevención de diseminación de enfermedades transfronterizas, como así también la necesidad de reforzar y promover los mecanismos de comunicación internacionales al respecto. En resumen, los resultados presentados en este trabajo sugieren que el movimiento legal de equinos desde Europa de acuerdo a la legislación vigente no impone un riesgo sustancial $\left(\mathrm{PP}_{\mathrm{VPEA}}<0,01\right)$ para la introducción de VPEA a los países del cono sur sudamericano y que, eventualmente, un test serológico negativo y certificaciones de libre en el establecimiento de origen serian medidas de mitigación suficientes para animales que se encontraban en tránsito al momento de detectarse un brote de PEA en el país origen del movimiento.

\section{REFERENCIAS}

MacLachlan NJ, AJ Guthrie. 2010. Re-emergence of bluetongue, African horse sickness, and other Orbivirus diseases. Vet Res 41, 35.

Mellor PS. 1993. African horse sickness: transmission and epidemiology. Vet Res 24, 199-212.

Mellor PS, C Hamblin. 2004 African horse sickness. Vet Res 35, 445-466.

Ministerio de Agricultura, Pesca y Alimentación. 2003. Índice del Estudio y Caracterización del Sector Equino en España. Ministerio de Agricultura, Pesca y Alimentación, Madrid, España.

Ministerio de Medio Ambiente y Medio Rural y Marino. 2011. Manual práctico de operaciones en la lucha contra la Peste Equina Africana (PEA). Ministerio de Medio Ambiente y Medio Rural y Marino, Madrid, España.

OIE, Organización Mundial de Sanidad Animal. 2013a . Análisis del riesgo asociado a las importaciones. En: OIE (ed). Código Sanitario para los Animales Terrestres. Vol. I. 22 a ed. OIE, Paris, Francia, Pp 99-104.

OIE, Organización Mundial de Sanidad Animal. 2013 ${ }^{\mathrm{b}}$. Infección por el Virus de la Peste Equina. En: OIE (ed). Código Sanitario para los Animales Terrestres. Volumen II. $22^{\mathrm{a}}$ ed. OIE, Paris, Francia, Pp 229-238.

Sánchez-Vizcaíno JM. 2004. Control and eradication of African horse sickness with vaccine. Dev Biol 119, 255-258.

Scacchia M, R Lelli, A Peccio, T Di Mattia, RS Mbulu, AL Hager, F Monaco, G Savini, A Pini. 2009. African horse sickness: a description of outbreaks in Namibia. Vet Ital 45, 265-274. 
